\title{
A Method of Image Enhancement Based on Non-sharpening Filtering and High Filtering
}

\author{
Lihua Sun ${ }^{\mathrm{a}}$, Enliang Zhao ${ }^{\mathrm{b},}$ * \\ Shenyang Jianzhu University, Shenyang, 110168, China

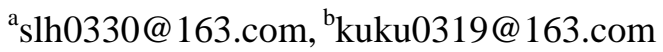 \\ *Corresponding author
}

Keywords: Image Enhancement, Non-sharpening Filtering, High Filtering

\begin{abstract}
This paper proposes a new method of image enhancement. Firstly, the image is sharpened by Laplace operator, and then the low frequency part of the image is removed by non-sharpening filtering, the high frequency part of the image is retained which will obtain a high pass image. At last the high filtering is made to enhance the image. The method is applied to numerical experiments. Experimental results show that proposed method can not only remove the low frequency part of the image, preserve the high frequency part of the image, but also improve the definition of the image. It is an effective method of image enhancement.
\end{abstract}

\section{Introduction}

Image enhancement has been widely used in space, medical images, military reconnaissance, fingerprint identification, satellite image processing and other fields [1-4]. The useful information is enhanced in the image. It can be a distortion of the process, in order to improve the visual effect of image. For a given image, it emphasizes the image of the whole or local characteristics which will make clearer image or emphasize certain interest characteristics, and expand the differences in the characteristics of image between different objects. At the same time indifferent feature is restrained so that it will improve image quality and abundant information, strengthen image interpretation and recognition in order to meet the needs of some special analysis. It is usually subjective for the results of image enhancement. Most of them rely on empirical accumulation method to process the images in practical applications in order that it can achieve the purpose of enhancement. It is also difficult to enhance the quantification standard for the image.

In the same neighborhood, enhancement algorithm of local contrast can enhance the details of the image by enhancing the pixels that are higher than the mean and weaken the pixels that are lower than the mean. However, the algorithm still processes the whole image which will make larger change for the image. It is not the best way to deal with the specific area. The enhancement algorithm of local contrast effectively enhances the local details. Because the amplification coefficient is not adjustable in the process of the local enhancement, it can not adjust the different regions at the same time it enhances the whole image. It is not ideal for the image with uneven contrast. We will dynamically adjust the amplification factor by the local standard deviation so that the region with small contrast is greatly enhanced, while the region with large contrast is less enhanced. The algorithm on adaptive local contrast enhancement introduces the local standard deviation in the amplification coefficient. It automatically adjusts the amplification coefficient according to the local standard deviation in the process of local enhancement at the same time it takes into account the different images in the contrast area when the image is enhanced.

This paper proposes a new method of image enhancement. At first, Laplace operator is used to sharpen the image. The low frequency part of the image is removed by non-sharpening filtering, the high frequency part of the image is retained which will obtain a high pass image. At last the image is enhanced by the high filtering. 


\section{Traditional Method of Image Enhancement}

The methods of traditional image enhancement can be divided into two types: image enhancement in the spatial domain and image enhancement in the frequency domain. The spatial domain refers to the set of pixels that constitute the image. The spatial domain image enhancement directly calculates and processes the pixel gray values in the image, such as grayscale transformation, histogram equalization, image spatial smoothing and sharpening, pseudo color processing, etc. Image enhancement in the frequency domain operates the spectral components of the image after Fourier transform, and then the inverse Fourier transform is used to get the desired results, such as low pass filtering technology, high pass filtering technology, band pass and band stop filtering, homomorphic filtering and so on. In order to adapt to the local characteristics of the image, image enhancement based on local transformation arises at the same, such as local histogram equalization [5], contrast constrained adaptive histogram equalization [6], and local statistical characteristics [7]. At present the image processing is combined with some subjects, which better overcomes the shortcomings of traditional transform enhancement technology a certain extent [8].

\section{Image Enhancement Method based on Non-Sharp Filtering and High Filtering}

Non-sharpening filtering is also called a non-sharpening mask. It refers that a non-sharpening or smooth image is subtracted from a original image in order to enhance the edge and other details of the image [9]. Let $f(x, y)$ represent the original image, $f_{s}(x, y)$ express the smooth image that obtained from the output image and $g(x, y)$ express the output image that has been processed by non-sharpening filtering. Thus we can use the formula (1) to denote the non-sharpening filtering.

$$
g(x, y)=f(x, y)-f_{s}(x, y)
$$

The essence of image smoothing is actually a low pass filtering, which subtracts a smooth image from the original image. That is to say, it removes the low frequency components of the original image, and retains the high frequency part of the original image, so that we can get a high pass image.

The image is processed by non-sharpening filtering which can remove the low frequency part and retain the high frequency part of the image. A high pass image is obtained. If we add the high pass image which is processed to the original image, we can keep the general picture of the original image, and at the same time, we can highlight the details of the edge and so on. It can also be expressed in another way, that is to multiply the original image by a ratio coefficient $A$, and at the same time, the high pass image is multiplied by a ratio coefficient. The enhanced image can be achieved by adding the two together. We can use the formula (2) to represent the high filtering.

$$
f_{h b}(x, y)=A f(x, y)+K g(x, y)
$$

Where $f_{h b}(x, y)$ is the output image that is processed by the high filtering, $g(x, y)$ is a high pass image of the input image $f(x, y)$, or it can be the result of non-sharpening mask. $A$ and $K$ are two proportionality coefficients that satisfy $A \geq 0,0 \leq K \leq 1$. When the value of $K$ is between 0.2 and 0.7 , the effect of high filtering is ideal. When the value $A$ is large enough, the sharpening effect of the image is relatively weakened, which makes the output image with the constant times of the input image is close. Figure 1 is several common templates for Laplace high filtering as the time $A$ is equal to 1 and $K$ equal to 1 .

$$
\left[\begin{array}{ccc}
0 & -1 & 0 \\
-1 & 5^{*} & -1 \\
0 & -1 & 0
\end{array}\right]
$$

(a)

$$
\left[\begin{array}{lll}
-1 & -1 & -1 \\
-1 & 9^{*} & -1 \\
-1 & -1 & -1
\end{array}\right]
$$

(b)

$$
\left[\begin{array}{ccc}
1 & -2 & 1 \\
-2 & 5^{*} & -2 \\
1 & -2 & 1
\end{array}\right]
$$

Fig.1. The common templates for Laplace high filtering 


\section{Experimental Results and Discussion}

Using Matlab7.0 software is adopted to implement the algorithm. In the experiment, a image of $256 \times 256$ is taken as an example. Figure 3 is the image which is processed by non-sharpening filtering. Figure 4 is the image that is further processed by a high filtering on the basis of Figure 3. Figure 5 is a contrast curve of the gray value.
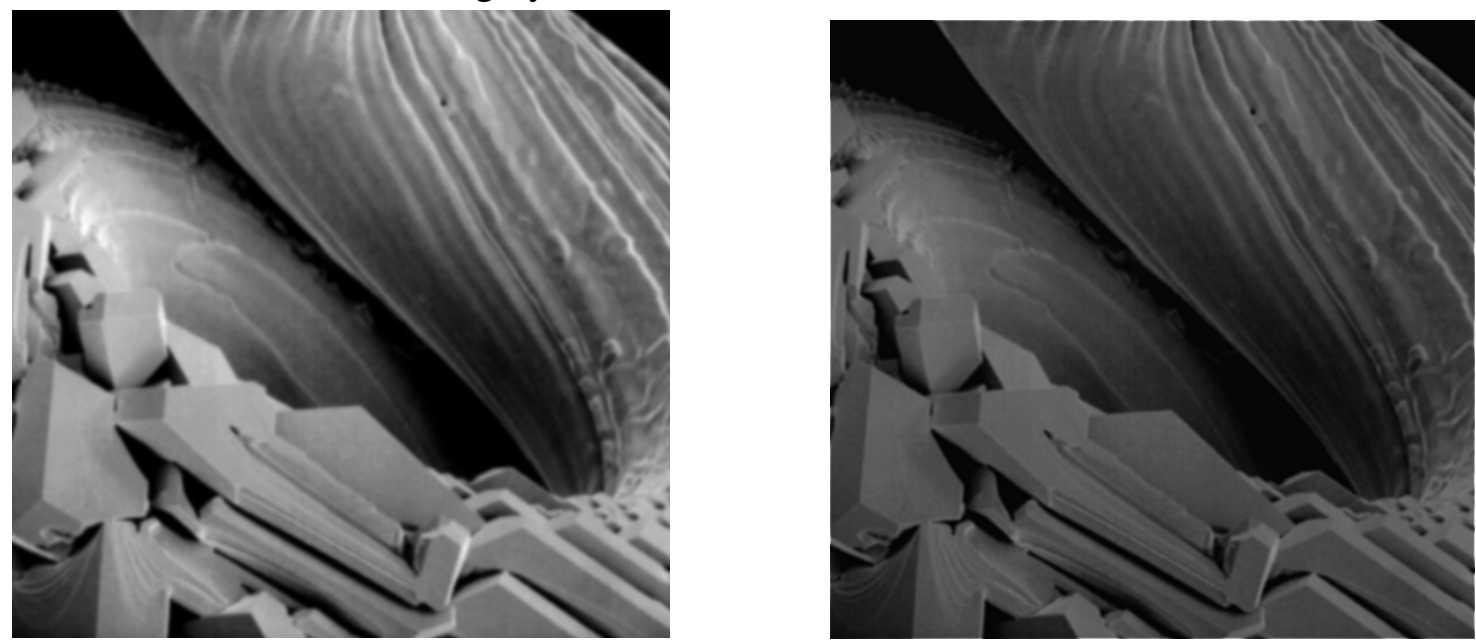

Fig.2. The Original image

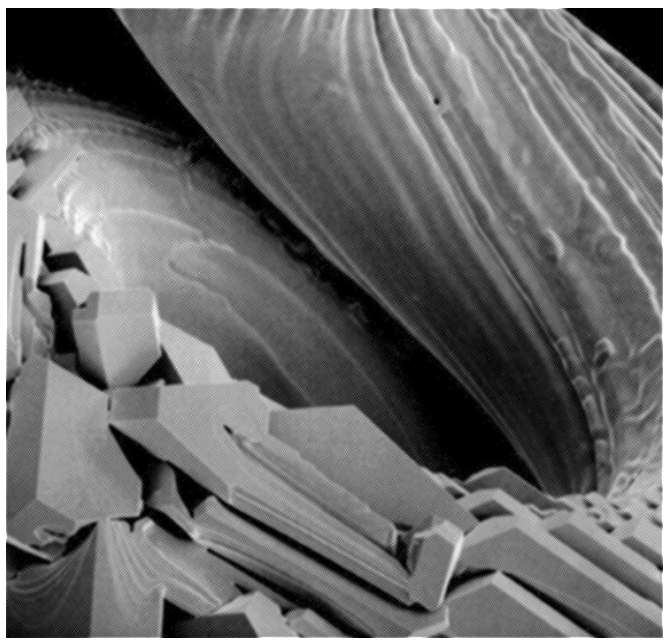

Fig.4. The Image processed by high filtering

Fig.3. The image processed by non-sharpening filtering

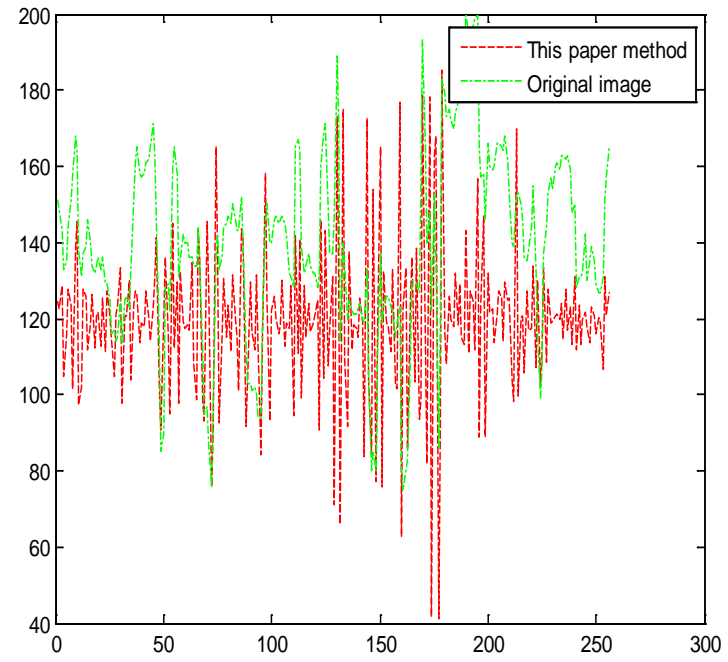

Fig.5. The contrast curve of gray value

The experimental results show that the image that is enhanced by the proposed method is clearer. It has a significant effect on the image enhancement. From the comparison curve of the gray value, we can see that the filtering can enhance the high frequency part of the image, restrain the low frequency part of the image, which may achieve the image enhancement.

\section{Conclusion}

A new method of image enhancement is proposed in this paper. Firstly, the image is sharpened by Laplace operator, and then the low frequency part of the image is removed by non sharpening filtering, the high frequency part of the image is retained which will obtain a high pass image. At last the high filtering is made to enhance the image. The method is applied to numerical experiments. Experimental results show that proposed method can not only remove the low frequency part of the image, preserve the high frequency part of the image, but also improve the definition of the image. It is an effective method of image enhancement. 


\section{References}

[1] Guo Yizheng, Jiao Pengpeng. Application of Matlab GUI in enhancement of low-quality fingerprint image [J]. Computer Technology and Development, 2013, 23(7): 230-232.

[2] He Shengzong, Liu Yingjie, Ma Yide, Song Wenqiang, Deng Haibo. Medical X-ray image enhancement based on PCNN image factorization [J]. Journal of Image and Graphics, 2011, 16(1):21-26.

[3] Kulkarni J, Patil B, Holambe R. Orientation feature for fingerprint matching [J]. Pattern Recognition, 2006, 39(8): 1551-1554.

[4] Jea T, Govindaraju V. A minutia-based partial fingerprint recognition system [J]. Pattern Recognition, 2005, 38(1): 1672-1684.

[5] Wang Zhiyun, Huang Mengwei, Hu Po. Image enhancement based on histograms and its realization with MATLAB [J]. Computer Engineering and Science, 2006, 28(2):54-56.

[6] Hu Zhengpin, Liu Bo. Dynamic partition image enhancement algorithm induced by optimal adaptive histogram specification function [J]. Journal of Yanshan University, 2009, 33(6):471-477.

[7] Popkin, T. Cavallaro, A. Hands, D. An Accurate and Efficient Method for Smoothly SpaceVariant Gaussian Blurring [J]. IEEE Transactions on Image Processing. 2010, 14(7): 139-142.

[8] Chen Meng. Study on ultrasonic image enhancement of ventricular septum [J]. Journal of Shangdong Medical College, 2012, 34(1): 76-77.

[9] He Dongjian. Digital Image Processing (Second Edition)[M]. Xian: Xidian University Press, 2009: 69-70. 